\title{
Low-Friction Self-Centering Droplet Propulsion and Transport Using a Leidenfrost Herringbone-Ratchet Structure
}

\author{
Linzi E. Dodd,,${ }^{1, *}$ Prashant Agrawal,${ }^{1}$ Matthew T. Parnell, ${ }^{1}$ Nicasio R. Geraldi, ${ }^{1}$ Ben B. Xu, ${ }^{1}$ \\ Gary G. Wells, ${ }^{1}$ Simone Stuart-Cole, ${ }^{2}$ Michael I. Newton, ${ }^{3}$ Glen McHale, ${ }^{1}$ and David Wood ${ }^{1}$ \\ ${ }^{1}$ Smart Materials and Surfaces Laboratory, Faculty of Engineering \& Environment, Northumbria University, \\ Newcastle upon Tyne NE1 8ST, United Kingdom \\ ${ }^{2}$ Reece Innovation, Armstrong Works, Scotswood Road, Newcastle upon Tyne NE15 6UX, United Kingdom \\ ${ }^{3}$ School of Science and Technology, Nottingham Trent University, Nottingham NG11 8NS, United Kingdom
}

(Received 29 March 2018; revised manuscript received 30 November 2018; published 27 March 2019)

\begin{abstract}
A fundamental limitation to the ability to transport sessile droplets is frictional forces arising from surface adhesion. This can be overcome by using the Leidenfrost effect on a heated substrate to levitate the droplet on a cushion of vapor. By structuring the surface under the droplet, the flow of vapor below the droplet can be controlled and this can be used to induce preferential droplet propulsion in a particular direction. However, while propulsion can be induced, the dramatic reduction in frictional forces leads to instability and it is difficult to control droplet motion when transporting droplets along a defined path. Here, we present a self-propulsion and self-centering concept using the principles of negative feedback to enable a droplet to be transported along a defined path. In our implementation, we use a combined herringbone and ratchet design, which provides the ability to control droplet position without compromising on speed. This intrinsic self-centering and correction via negative feedback offers the potential to design paths and tracks for droplets to follow, without the need for walls.
\end{abstract}

DOI: 10.1103/PhysRevApplied.11.034063

\section{INTRODUCTION}

The Leidenfrost effect, discovered in 1756 [1], occurs when a liquid droplet is placed on a substrate at a temperature significantly higher than the liquid's boiling point. The droplet then levitates (Fig. 1), on a cushion of its own vapor, which insulates the droplet and significantly reduces the evaporation rate $[2,3]$. Recently, there has been a substantial amount of interest in frictionless transport, using the Leidenfrost effect to levitate and propel droplets [4-13]. However, these previous works do not simultaneously control the position of the droplet or allow the droplet to change direction without the need for physical barriers.

Various different methods of controlling droplet motion using the Leidenfrost effect have been attempted [4-8], including structuring the substrate to encourage propulsion [13]. In 2006, Linke et al. were the first to show a self-propelled Leidenfrost droplet on a ratchet surface

*linzi.dodd@northumbria.ac.uk

Published by the American Physical Society under the terms of the Creative Commons Attribution 4.0 International license. Further distribution of this work must maintain attribution to the author(s) and the published article's title, journal citation, and DOI.
[9]. Dupeux et al. discuss the mechanism involved in ratchet propulsion [10] and Cousins et al. have successfully trapped a droplet in a circular ratchet structure [11]. In 2016, D. Soto et al. showed that propulsion can occur on a herringbone-structured surface [12].

One serious limitation of all of these previous studies is that droplets do not stay on the intended paths without the use of barriers. This is because the extreme reduction in friction caused by levitating the droplet on a cushion of vapor allows small perturbations to create instability in directional motion. In our work, self-centering droplet motion is created by texturing the substrate and so removing the need for physical boundaries. Guided by the principles of negative feedback, we implement substrate designs that enable the self-correction of motion and have the potential to transform the use of the Leidenfrost effect for droplet control. Once self-centering has been achieved, the direction of droplet motion can be varied and controlled as required for any given application.

A pure ratchet structure provides high acceleration and terminal velocity, but the droplets are not confined to the surface and so often travel off the edges of the block. The effect is enhanced if temperature variations occur or if the sizes of the droplet and of the ratchets are comparable [14]. To achieve propulsion on a herringbone surface, the droplet must be on the center-line herringbone [12]. The 


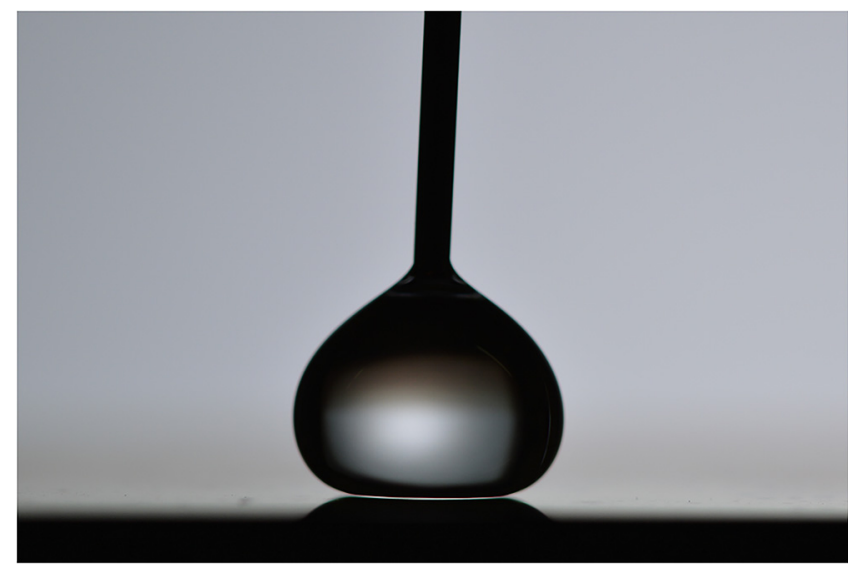

FIG. 1. A droplet levitating on its vapor layout on a superheated surface due to the Leidenfrost effect.

centered propulsion of a herringbone design and the directional flow of a ratchet surface are combined in our work to create self-centered droplet propulsion without compromising on the droplet velocity. Our specific designs illustrate the general principle of using negative feedback and Leidenfrost levitation to achieve stable low-friction motion along user-defined pathways.

\section{METHODS}

\section{A. Ratchet and herringbone-ratchet fabrication details}

CZ121 brass blocks were machined with fixed-angle tools to provide right-angled triangles of differing heights and therefore ratchet angles $(\beta), 1 \mathrm{~mm}$ wide, which were repeated to create a sawtooth pattern at $90^{\circ}$ to the long edge for the ratchet block, as seen in Fig. 2(a), and at a $45^{\circ}$ angle $(\alpha)$ to the long edge for the herringbone ratchet. For the herringbone ratchet, the patterns were reproduced in mirror image so that when two blocks were brought into contact, the herringbone-ratchet shape was created, as seen in Fig. 2(c).

The water used in the experiments was preheated to just below the boiling point prior to deposition on the ratchet or herringbone-ratchet surface. The droplets were deposited by dispensing the droplet on the surface and then being held briefly, prior to the syringe tip being released, to ensure that no momentum was added to the experiment from the dispensing procedure and to minimize any jetting effects. Wider syringe tips were used for the larger droplet volumes.

A typical ratchet design can be seen in Fig. 3. Preliminary ratchet-structure analysis involved testing droplets on ratchet structures with different heights of right-angled sawtooth triangles, including $0.26,0.47,1.0$, and $2.0 \mathrm{~mm}$ heights, all with a $1 \mathrm{~mm}$ width, to provide different ratchet tooth angles $\left(\beta=15^{\circ}, 25^{\circ}, 42^{\circ}\right.$, and $63^{\circ}$, respectively). These experiments showed that the highest-speed ratchet geometry was the $0.26 \mathrm{~mm}$ high (or $15^{\circ}$ ) ratchet, with a $40 \mu 1$ droplet, tested at $250^{\circ} \mathrm{C}$, as can be seen in Table I. Furthermore, for droplet sizes above $100 \mu 1$, both the $15^{\circ}$ and $25^{\circ}$ ratchets provide comparable droplet speeds, with the $25^{\circ}$ ratchet providing droplet speeds that are independent of volume within the range 100-600 $\mu$ l. Droplet propulsion was unsuccessful on the 2-mm-high ratchet.

\section{B. The model for droplet motion driven by the ratchet portion of the herringbone ratchet}

To understand how the self-centering motion works, it is necessary to characterize the motion of droplets on the ratchet portion of the herringbone ratchet and to understand how the driving force depends on the design of the ratchet angle. The motion of a given liquid droplet depends on the substrate design, the substrate temperature, and the size of the droplet. It is also sensitive to local temperature variations of the substrate and the deformation of the droplet over the substrate. Here, we provide an approach that (i) quantitatively describes the motion of a droplet when deposited off-center, (ii) provides an understanding of key design features on the ratchet portion of the structure, and (iii) can then be used to establish design principles for self-centering droplet movement on the herringbone ratchet.

In the literature, several mechanisms have been suggested to explain the self-propulsion of droplets due to substrate asymmetry, such as a jet-thrust effect [13],
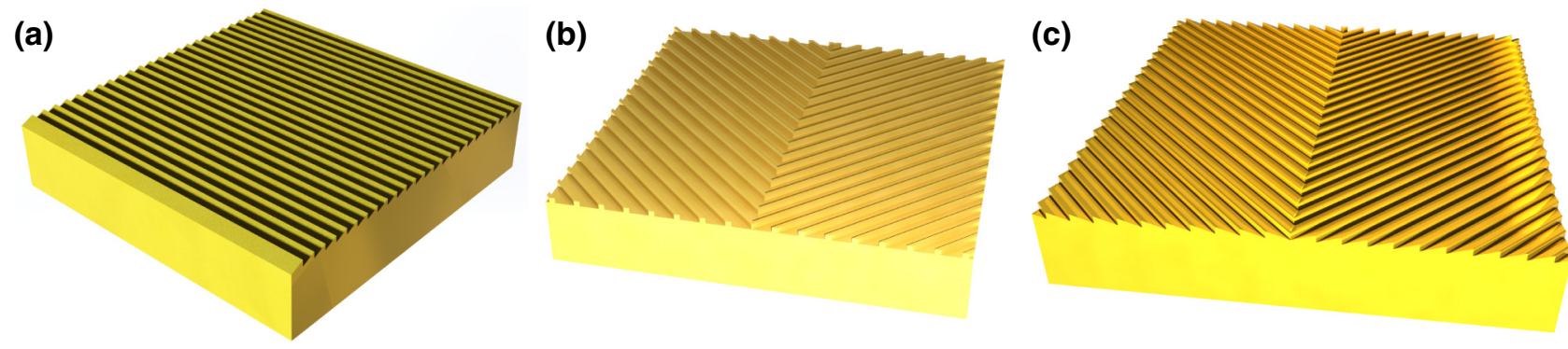

FIG. 2. Renderings of (a) a typical ratchet structure, (b) a typical herringbone structure, and (c) a proposed herringbone-ratchet structure with a ratchet pattern rotated at an angle of $\alpha=45^{\circ}$ from the center line. 

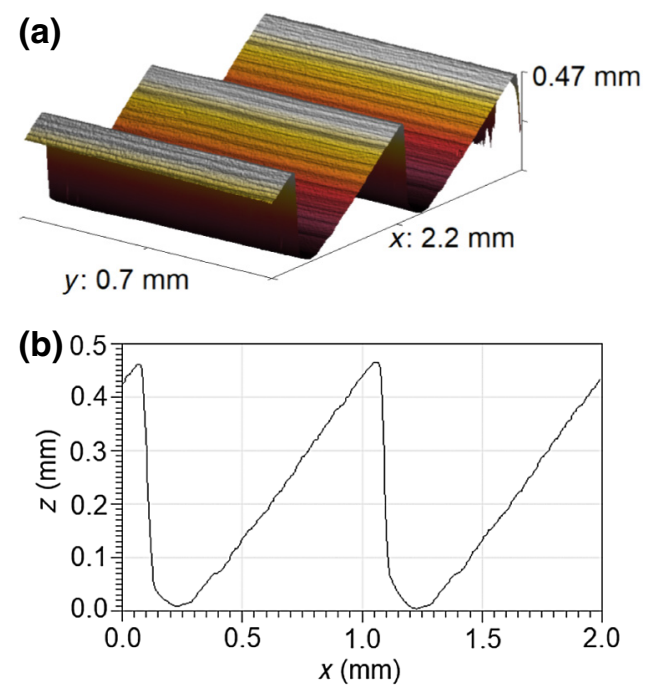

FIG. 3. The profile of a typical ratchet design, measured using an optical profiler (Bruker ContourGT): (a) the threedimensional (3D) profile; (b) the two-dimensional (2D) cross section, showing the dimensions of the ratchet structure.

thermal creep flows [15], temperature-difference-induced Marangoni flows [16], and vapor rectification by the substrate geometry $[9,10,12]$. However, previous experimental $[12,17,18]$ and numerical $[10-12,17,18]$ works have demonstrated that the viscous-stress-induced propulsion due to vapor rectification is dominant. Most notable is the work by Soto et al. [12], which employs rectangular crosssection grooves to evacuate vapor asymmetrically when arranged in a herringbone pattern. The use of such locally symmetric tooth profile negates the effect of any gradients induced due to thermal creep or Marangoni flows. Although Marangoni-driven flows have also been highlighted as a possible self-propulsion mechanism [16,19], recent studies have shown that internal flows homogenize the temperature in the drop [20]. Therefore, rotation due to Marangoni flows might be present but has a marginal role in self-propulsion. We therefore believe it is reasonable to assume that shear stress induced due to vapor rectification is the dominant propulsion mechanism.

A droplet moving on the ratchet portion of the substrate is driven by a propulsion force originating from the vapor and an inertial resistance acting on the liquid-vapor interface that originates from the minor shocks from the textured substrate [13]. The viscous resistance from the vapor flow is negligible compared to the inertial resistance [13]. As a result, for a droplet far from the center line on the substrate at a given temperature and of specific ratchet angle design, its equation of motion in the direction of the ratchet slope can be written as follows:

$$
m \dot{v}=F_{p}-c_{i} v^{2}
$$

where, $F_{p}$ is the propulsion force on the droplet, $m$ is the mass of the droplet, $v$ is the droplet's velocity, and $c_{i}$ is a numerical coefficient representing the inertial resistance offered by the ratchet. The parameter $c_{i}$ will depend on the ratchet design, the temperature, the droplet's volume and shape, and the deformation of the droplet over the ratchet grooves. By using Fig. 1, we assume that the inertial resistance for drop movement parallel to the ratchets is relatively small [12]. For a droplet released from rest, the solution to Fig. 1 is as follows:

$$
v=v_{t} \tanh (t / \tau)
$$

Here, $v_{t}=\sqrt{F_{p} / c_{i}}$ is the terminal velocity of the droplet and $\tau=m / \sqrt{c_{i} F_{p}}$ is the relaxation time and gives an estimate of the droplet's acceleration; the time taken by the droplet to achieve $99 \%$ terminal velocity is given as $t=$ $2.64 \tau$. Equation (2) describes the motion of a droplet that, at time $t=0$, initially accelerates with $v_{t} / \tau$ and then eventually achieves a constant velocity of $v_{t}$. For a given droplet volume, the motion of the droplet can be characterized by deducing $F_{p}$ and $c_{i}$ from experimental droplet motion data. To do so, the displacement $(s)$ of the droplet with time is obtained for the desired droplet volume and substrate temperature on the design of interest and fitted to the integral of Eq. (2), i.e.:

$$
s=v_{t} \tau \ln [\cosh (t / \tau)]
$$

Figure 4 shows the droplet displacement data for different droplet volumes on the ratchet-only substrate with $\beta=25^{\circ}$ at a substrate temperature of $250^{\circ} \mathrm{C}$. As seen by the fit curves in Fig. 4, Eq. (3) accurately describes each set of displacement data curves and provides estimates of $v_{t}$ and $\tau$, and hence the propulsion force $F_{p}$ and the coefficient $c_{i}$ representing the inertial resistance offered by the ratchet, for each case. The inset of Fig. 4 shows the droplet speeds, using $v_{t}$ and $\tau$ in Eq. (2), deduced from the experimental data.

\section{Simulations of the viscous propulsion force due to the ratchet portion of the herringbone ratchet}

To better understand the design of the herringbone ratchet, we use numerical simulations of the vapor flow over a ratchet to calculate the viscous propulsion force and its dependence on the ratchet geometry. The propulsion force obtained from the simulations is denoted as $F_{s}$ and is assumed to provide an approximation to $F_{p}$ as defined in the previous section.

A 3D single-phase laminar-flow incompressible numerical model (using Ansys 17.1) is employed, to simulate the flow of vapor from a stationary evaporating droplet (with a circular base) over the ratchet, as depicted in Fig. 5(b). The simulation domain models the vapor phase 
TABLE I. A summary of the features and speeds of different propulsion structures.

\begin{tabular}{|c|c|c|c|c|c|c|c|}
\hline $\begin{array}{c}\text { Design } \\
\text { structure }\end{array}$ & $\begin{array}{c}\text { Feature } \\
\text { height }(\mathrm{mm})\end{array}$ & $\begin{array}{c}\text { Ratchet angle, } \\
\beta \text { (degrees) }\end{array}$ & $\begin{array}{l}\text { Temperature } \\
\left( \pm 5^{\circ} \mathrm{C}\right)\end{array}$ & $\begin{array}{l}\text { Droplet } \\
\text { volume } \\
(\mathrm{ml})\end{array}$ & $\begin{array}{c}\text { Experimental } \\
\text { droplet maximum } \\
\text { velocity }\left(\mathrm{mm} \mathrm{s}^{-1}\right)\end{array}$ & $\begin{array}{l}\text { Fitted terminal } \\
\text { velocity, } v_{t} \\
\left(\mathrm{~mm} \mathrm{~s}^{-1}\right)\end{array}$ & $\begin{array}{l}\text { Fitted } \\
\text { relaxation } \\
\text { time, } \tau(\mathrm{s})\end{array}$ \\
\hline \multirow[t]{15}{*}{ Ratchet } & 0.26 & 15 & 250 & 0.04 & $350 \pm 10$ & $350 \pm 10$ & $0.33 \pm 0.04$ \\
\hline & 0.26 & 15 & 250 & 0.10 & $315 \pm 5$ & $340 \pm 20$ & $0.62 \pm 0.04$ \\
\hline & 0.26 & 15 & 250 & 0.20 & $240 \pm 10$ & $300 \pm 20$ & $0.83 \pm 0.07$ \\
\hline & 0.26 & 15 & 250 & 0.40 & $250 \pm 30$ & $220 \pm 40$ & $0.70 \pm 0.20$ \\
\hline & 0.26 & 15 & 250 & 0.60 & $180 \pm 20$ & $140 \pm 40$ & $1.10 \pm 0.50$ \\
\hline & 0.47 & 25 & 250 & 0.04 & $180 \pm 30$ & $160 \pm 20$ & $0.27 \pm 0.08$ \\
\hline & 0.47 & 25 & 250 & 0.10 & $250 \pm 20$ & $260 \pm 30$ & $0.50 \pm 0.10$ \\
\hline & 0.47 & 25 & 250 & 0.20 & $250 \pm 30$ & $260 \pm 20$ & $0.46 \pm 0.06$ \\
\hline & 0.47 & 25 & 250 & 0.40 & $270 \pm 20$ & $270 \pm 20$ & $0.56 \pm 0.08$ \\
\hline & 0.47 & 25 & 250 & 0.60 & $250 \pm 40$ & $270 \pm 30$ & $0.60 \pm 0.20$ \\
\hline & 1.00 & 45 & 250 & 0.04 & $120 \pm 20$ & $127 \pm 6$ & $0.29 \pm 0.03$ \\
\hline & 1.00 & 45 & 250 & 0.10 & $130 \pm 30$ & $200 \pm 20$ & $0.90 \pm 0.10$ \\
\hline & 1.00 & 45 & 250 & 0.20 & $160 \pm 30$ & $250 \pm 40$ & $1.10 \pm 0.20$ \\
\hline & 1.00 & 45 & 250 & 0.40 & $180 \pm 20$ & $250 \pm 20$ & $0.90 \pm 0.20$ \\
\hline & 1.00 & 45 & 250 & 0.60 & $180 \pm 10$ & $240 \pm 40$ & $1.00 \pm 0.20$ \\
\hline \multirow[t]{20}{*}{ Herringbone ratchet } & 0.26 & 15 & 250 & 0.04 & $330 \pm 20$ & $290 \pm 20$ & $0.21 \pm 0.02$ \\
\hline & 0.26 & 15 & 250 & 0.10 & $330 \pm 20$ & $280 \pm 20$ & $0.27 \pm 0.02$ \\
\hline & 0.26 & 15 & 250 & 0.20 & $300 \pm 10$ & $290 \pm 10$ & $0.46 \pm 0.02$ \\
\hline & 0.26 & 15 & 250 & 0.40 & $250 \pm 20$ & $200 \pm 20$ & $0.20 \pm 0.02$ \\
\hline & 0.26 & 15 & 250 & 0.60 & $240 \pm 10$ & $200 \pm 10$ & $0.35 \pm 0.02$ \\
\hline & 0.26 & 15 & 320 & 0.04 & $220 \pm 10$ & $210 \pm 10$ & $0.59 \pm 0.03$ \\
\hline & 0.26 & 15 & 320 & 0.10 & $200 \pm 10$ & $173 \pm 10$ & $0.45 \pm 0.03$ \\
\hline & 0.26 & 15 & 320 & 0.20 & $210 \pm 10$ & $190 \pm 10$ & $0.56 \pm 0.03$ \\
\hline & 0.26 & 15 & 320 & 0.40 & $180 \pm 10$ & $153 \pm 10$ & $0.68 \pm 0.03$ \\
\hline & 0.26 & 15 & 320 & 0.60 & $150 \pm 20$ & $130 \pm 20$ & $0.8 \pm 0.1$ \\
\hline & 0.90 & 42 & 250 & 0.04 & $200 \pm 20$ & $190 \pm 20$ & $0.42 \pm 0.05$ \\
\hline & 0.90 & 42 & 250 & 0.10 & $210 \pm 20$ & $190 \pm 20$ & $0.46 \pm 0.05$ \\
\hline & 0.90 & 42 & 250 & 0.20 & $220 \pm 20$ & $200 \pm 20$ & $0.38 \pm 0.04$ \\
\hline & 0.90 & 42 & 250 & 0.40 & $220 \pm 20$ & $180 \pm 20$ & $0.17 \pm 0.02$ \\
\hline & 0.90 & 42 & 250 & 0.60 & $250 \pm 20$ & $220 \pm 20$ & $0.31 \pm 0.03$ \\
\hline & 0.90 & 42 & 320 & 0.04 & $240 \pm 10$ & $190 \pm 10$ & $0.29 \pm 0.02$ \\
\hline & 0.90 & 42 & 320 & 0.10 & $240 \pm 20$ & $220 \pm 20$ & $0.38 \pm 0.04$ \\
\hline & 0.90 & 42 & 320 & 0.20 & $280 \pm 20$ & $240 \pm 20$ & $0.31 \pm 0.03$ \\
\hline & 0.90 & 42 & 320 & 0.40 & $290 \pm 10$ & $260 \pm 10$ & $0.29 \pm 0.02$ \\
\hline & 0.90 & 42 & 320 & 0.60 & $300 \pm 20$ & $280 \pm 20$ & $0.41 \pm 0.03$ \\
\hline
\end{tabular}

around the droplet and the substrate, as shown in Fig. 5(b) and 5(c).

The evaporating liquid-vapor interface (boundary 1) is modeled as an air inlet supplying vapor at constant velocity (in the $z$ direction) over the substrate. The air-flow velocity is obtained from experiments using the evaporation rate of a droplet of given volume. Given the droplet volumes used in the experiments (the drop size is greater than the capillary length $l_{c}$ ), the droplet radius near the substrate is estimated by assuming a puddle shape with height $2 l_{c}$ [21]. The substrate boundary (boundary 4) is assigned a no-slip stationary-wall condition. The distance between the liquid-vapor interface of the droplet and the substrate $(h)$ is taken as $50 \mu \mathrm{m}$, which is representative of the scale of the vapor-layer thickness of droplets in the Leidenfrost state [3]. This thickness is also a computational constraint with regard to the large computation time needed for smaller thicknesses. The boundaries representing the liquid-vapor interface around the droplet (boundary 3) are set as freeslip boundaries, which neglects any shear forces due to the flow inside the droplet. The other boundaries are set at zero relative pressure, which simulates the surrounding atmosphere (boundary 2). The flow properties of the vapor are taken for steam at $100^{\circ} \mathrm{C}$. The propulsion force on the droplet is calculated by integrating the shear stress on the liquid-vapor interface of the droplet (near the substrate) over its base area $A$ :

$$
F_{s}=\int_{A} \mu\left(\frac{\partial u_{x}}{\partial z}+\frac{\partial u_{y}}{\partial z}\right) d A
$$




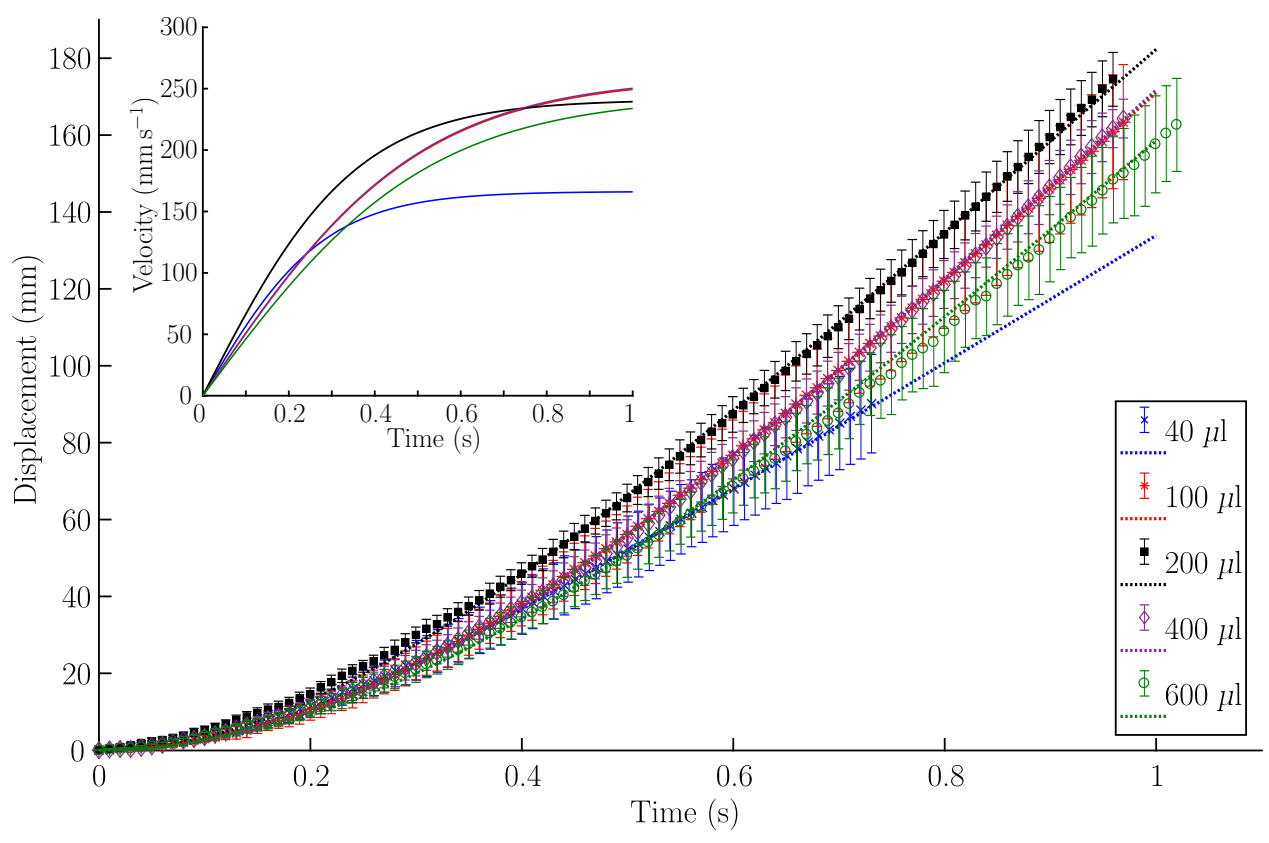

FIG.

4. Experimentally observed displacement profiles of water droplets of different volumes on a ratchet-only substrate with $\beta=25^{\circ}$ at $250^{\circ} \mathrm{C}$. The dotted lines are the fit curves of Eq. (3) on the experimental data, which are used to obtain $v_{t}$ and $\tau$ for each droplet volume. The inset shows the velocity vs time curves, represented by Eq. (2) using the values of $v_{t}$ and $\tau$.

Here, $\mu$ is the dynamic viscosity of the vapor, $u_{x}$ is the vapor velocity in the $x$ direction, and $u_{y}$ is the vapor velocity in the $y$ direction, as defined in Fig. 5. The mesh convergence analysis of the simulations is discussed in Appendix.

Figure 6 shows simulation results comparing the propulsion force $\left(F_{s}\right)$ on a stationary droplet $(V=600 \mu \mathrm{l})$ for different ratchet geometries. The asymmetry in the tooth geometry gives rise to a pressure gradient in the generated vapor layer. This pressure gradient leads to a viscositydominated propulsion force on the levitating droplet, in the $x$ direction $[9,10]$. As a consequence, the viscous propulsion force depends on the pressure gradient and the vapor-film thickness. As the tooth angle increases, the pressure difference between the tip and the tooth base increases, which implies an increase in the propulsion force. However, a decrease in the average vapor-layer thickness introduces a competing factor that decreases the

(a)

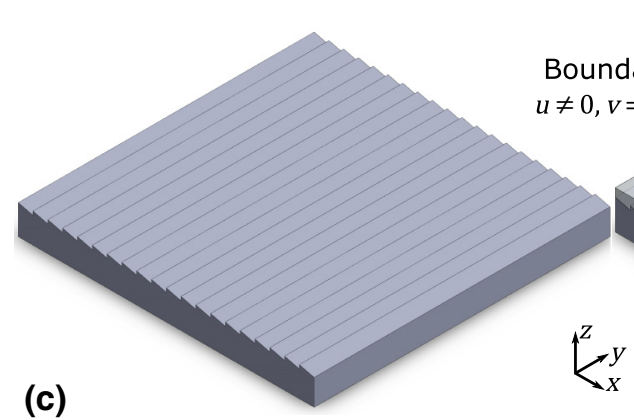

(b) Boundary 3: Free slip

propulsion force. Numerical simulations shown in Fig. 6 agree with the above explanation where, for smaller tooth angles, the increase in propulsion force with tooth angle, proportional to $\tan ^{3} \beta$, agrees with analytical formulations for $\beta<10^{\circ}[17,18]$. We also note that a $\cot \beta$ dependence appears to fit the higher angles, although there is no analytical model for this limit.

\section{Drop movement on a herringbone ratchet}

To simulate the movement of an off-center droplet, Eqs. (5) and (6) are used to track the $x$ and $y$ positions of the droplet at each time $t$ (using Matlab):

$$
m \dot{v}_{x}=F_{p} \sin \alpha-\frac{c_{i} v_{x}^{2} \sin \left(\alpha-\theta_{d}\right)}{\sin \alpha}
$$

FIG. 5. (a) A pictorial representation of a ratchet substrate. (b) The simulation domain of the

Boundary 2

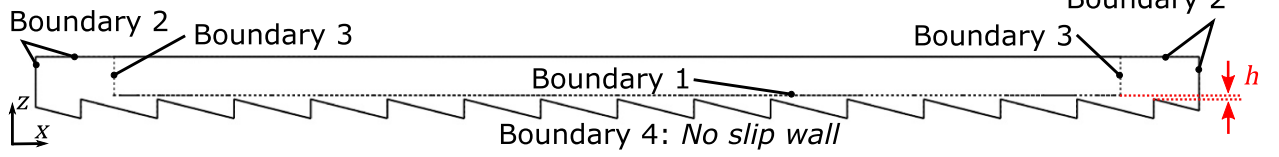
vapor above the substrate and the boundary conditions employed for simulating vapor flow from an evaporating droplet over the ratchet substrate. (c) The side view of the simulation domain and the boundary conditions. 


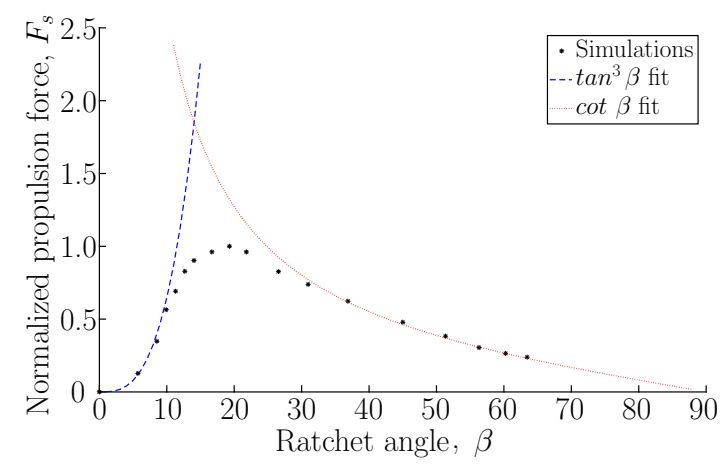

FIG. 6. Simulation results of vapor flow showing comparison of the propulsion force $\left(F_{s}\right)$ on a stationary droplet $(V=600 \mu 1)$ for different ratchet geometries. The propulsion shear force has been normalized by the maximum shear force, which occurs at a ratchet angle of approximately $19^{\circ}\left(F_{s}=690 \mu \mathrm{N}\right)$.

$$
m \dot{v}_{y}=F_{p} \cos \alpha-\frac{c_{i} v_{y}^{2} \sin \left(\alpha-\theta_{d}\right)}{\cos \alpha},
$$

where, in this coordinate system, $x$ is along the center line of the herringbone ratchet, $y$ is perpendicular to $x$, and $\alpha$ is the herringbone angle, which is the angle of the ratchet grooves with $x$. Here, $v_{x}$ is the droplet velocity in the $x$ direction, $v_{y}$ is the droplet velocity in the $y$ direction, and $\theta_{d}=\operatorname{atan}\left(v_{y} / v_{x}\right) ; \alpha-\theta_{d}$ represents the angle at which the droplet travels relative to the ratchet grooves. As the droplet is propelled by the ratchet part of the substrate, $F_{p}$ and $c_{i}$ represent the propulsion force and the inertial damping coefficient of the ratchet only, obtained from characterizing the motion on the ratchet portion of the substrate as described previously (Sec. II B).

The motion along the center line of the herringbone ratchet can be described by an equation of the form given by Eq. (1), with a solution in the form of Eq. (2) [and Eq. (3)]. This motion will be characterized by an inertial damping coefficient for droplet movement along the center line, $c_{c l}$, different to that obtained for motion purely on the ratchet portion of the substrate $c_{c l} \sim c_{i} \sin ^{3} \alpha$ [12]. By describing droplet motion using Eq. (2), we develop a common method for comparing the propulsion characteristics of different ratchet and herringbone-ratchet designs. Furthermore, by using numerical simulations in conjunction with experimental data, we are able to qualitatively understand the centering characteristics of herringbone ratchets, which aids in establishing their design principles.

\section{RESULTS AND DISCUSSION}

As mentioned in Sec. II D, to explore droplet movement on a herringbone-ratchet substrate, droplet motion on ratchet-only substrates are analyzed. These studies on the ratchets provide information about the propulsion characteristics of the ratchet (Sec. II), which, combined with the previously studied herringbone design [12], informs the implementation of a self-centering herringbone-ratchet design. The self-centering characteristics of a herringboneratchet design illustrate the concept of negative-feedback control of the droplet position in Leidenfrost levitation and transportation.

As described in Sec. II A, we find that the highest-speed experimental ratchet geometry results occur for $\beta=25^{\circ}$ : this structure is shown in Fig. 3. Video evaluation of ratchet structures has shown that the droplets travel at a terminal velocity of approximately $300 \mathrm{~mm} \mathrm{~s}^{-1}$, as can be seen in Fig. 4. As expected, the droplets move in the direction of the downward slope of the sawtooth triangles. Over the volume range that we consider, the droplets' terminal velocity shows a weak dependence on droplet volume. There is no centering of the droplets on this configuration of block. Furthermore, they reach a terminal velocity within approximately $0.4 \mathrm{~s}$, with smaller droplets accelerating to their terminal velocities more quickly than the larger droplets.

To achieve centering capabilities, we now consider the ability of the combined herringbone-ratchet structure [Fig. 2(c)], inspired by the herringbone structure previously investigated by Soto et al. [12], to provide negative feedback and Leidenfrost levitation to achieve stable low-friction motion along user-defined pathways.

\section{A. Droplet centering on a herringbone ratchet}

Video 1 shows droplets centering on a herringbone ratchet with a herringbone angle of $\alpha=45^{\circ}$ and a ratchet angle of $\beta=42^{\circ}$. It can be seen that a droplet deposited off center is propelled toward the center line. After a few underdamped oscillations about the center line, it self-centers and travels along the center line until reaching the end of the substrate. This experiment verifies the self-centering characteristics of the herringbone ratchet.

Another herringbone-ratchet design with $\alpha=42^{\circ}$ and a ratchet angle of $\beta=15^{\circ}$ is considered (Fig. 7). Based on simulations and experiments, this reduced ratchet angle provides a relatively higher propulsion force, as seen in

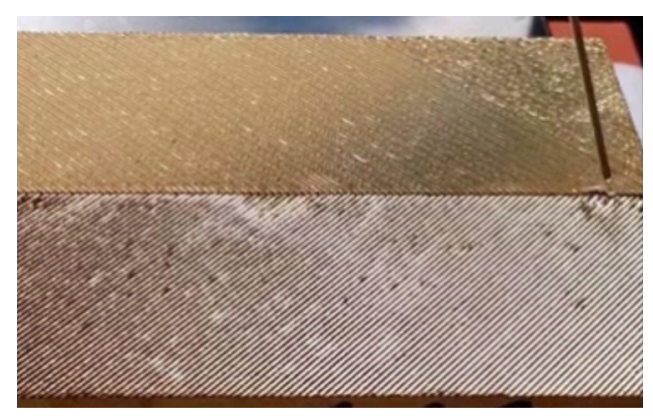

VIDEO 1. A video showing 1-mm-high by 1-mm-wide sawtooth triangles (ratchet angle $\beta=45^{\circ}$ ) in a herringbone-ratchet structure. 


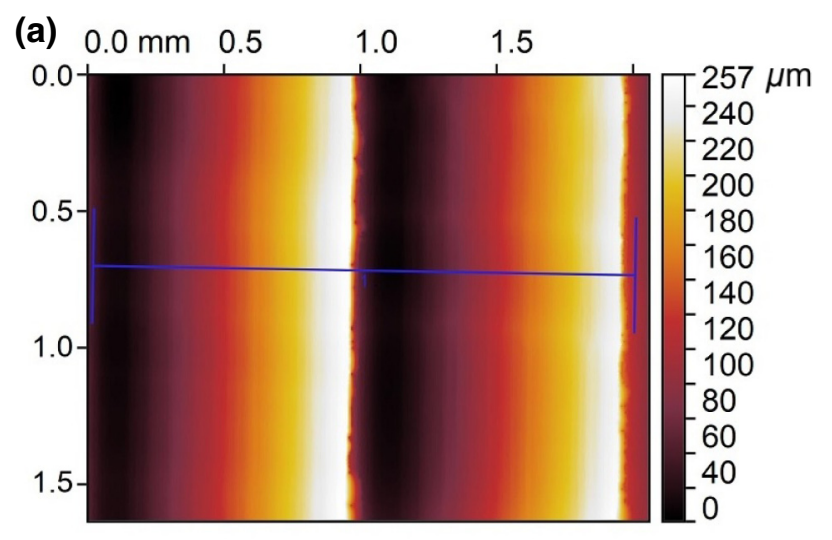

(b)

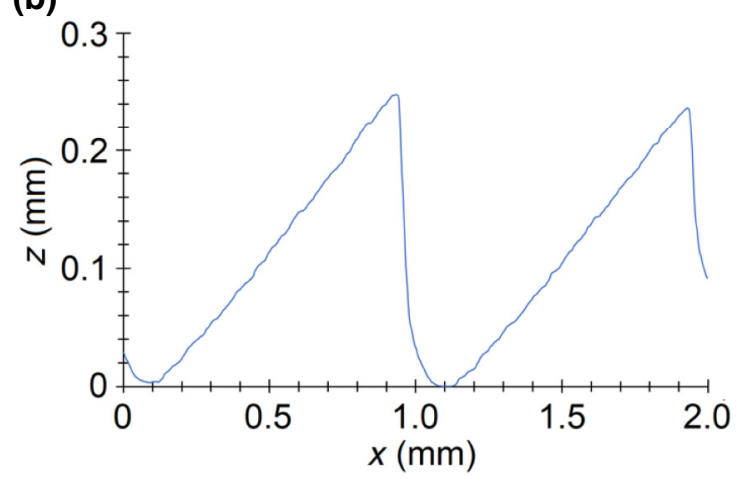

FIG. 7. The profile analysis of the reduced-height ratchet in a herringbone-ratchet design.

Fig. 6. Although a ratchet with the highest propulsion force is expected at a higher ratchet angle $\left(\beta=19^{\circ}\right)$, the aim is to provide a slight increase in velocity, but as a compromise with centering capability.

As seen in Videos 1 and 2, droplets deposited off center tend to overshoot and then correct repeatedly, due to negative feedback, resulting in an underdamped oscillation about the center line. The videos also show that the centering strength of the herringbone ratchet with ratchet angle $\beta=15^{\circ}$ is lower than that of a herringbone ratchet with $\beta=42^{\circ}$.

The difference in centering characteristics of the abovementioned designs can be understood by looking at the

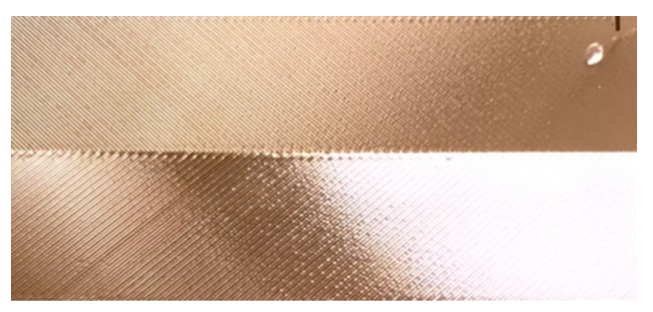

VIDEO 2. A video showing 0.26-mm-high by 1-mm-wide sawtooth triangles (ratchet angle $\beta=15^{\circ}$ ) in a herringboneratchet structure.

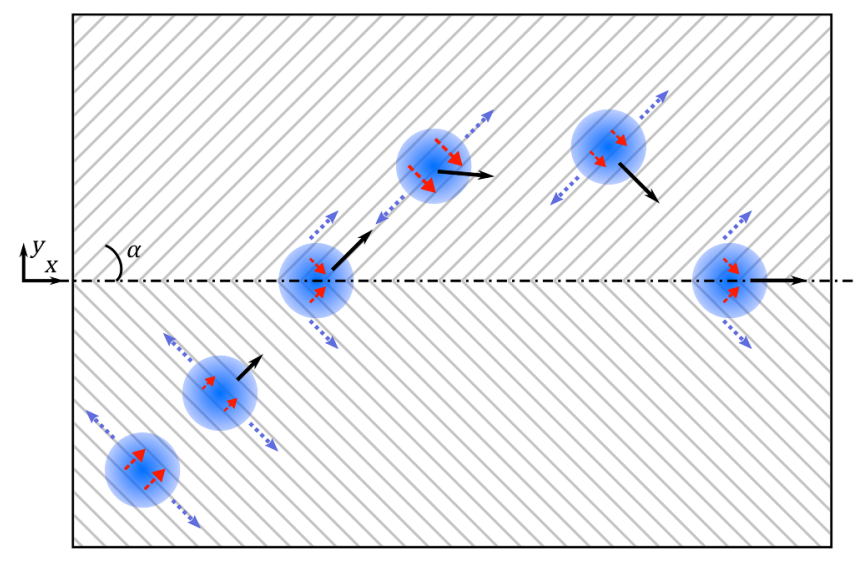

FIG. 8. A pictorial representation of droplet movement on a herringbone-ratchet structure, showing the air-flow direction (blue arrows), the force due to the ratchet geometry (red dotted arrows), and the velocity vector of the droplet (black solid arrow).

forces on a droplet at different instances on the herringbone ratchet. Consider a droplet deposited at position $|y| \ll R$ (where $R$ is the droplet radius) on a herringbone ratchet, as depicted in Fig. 8. At this position, the droplet movement is decided solely by the ratchet structure, through the propulsion force $\left(F_{p}\right)$ and the time to reach terminal velocity (indicated by $\tau$ ). As it crosses the center, the direction of the propulsion force changes and the droplet decelerates in $y$, i.e., the propulsion force now provides a restoring force along with the inertial resistance $\left(c_{i}\right)$. The distance the droplet travels before reversing toward the center depends on the droplet's inertia $\left(\rho R^{2} v^{2}\right)$ while crossing the center, the propulsion force $\left(F_{p}\right)$, and the inertial resistance from the ratchet $\left(c_{i} v^{2}\right)$. As the droplet spends a relatively short time near the center, the herringbone element of the design does not contribute to the centering capabilities. Once the droplet stabilizes in the region $|y|<R$, the herringbone element also contributes toward the propulsion in the $x$ direction.

Assuming that the droplet attains its terminal velocity before reaching the center line, the distance in $x$ at which the droplet centers, from its point of deposition, is termed the "centering distance" $d_{c}$ and can be expressed as follows:

$$
d_{c} \propto \frac{\rho R^{2} v_{t}^{2}}{F_{p}+c_{i} v_{t}^{2}} .
$$

On rearranging, Eq. (7) becomes

$$
d_{c} \propto \frac{1}{F_{p} / v_{t}^{2}+c_{i}} .
$$

Equation (8) assumes that the droplet reaches its terminal velocity associated with motion on the ratchet portion 


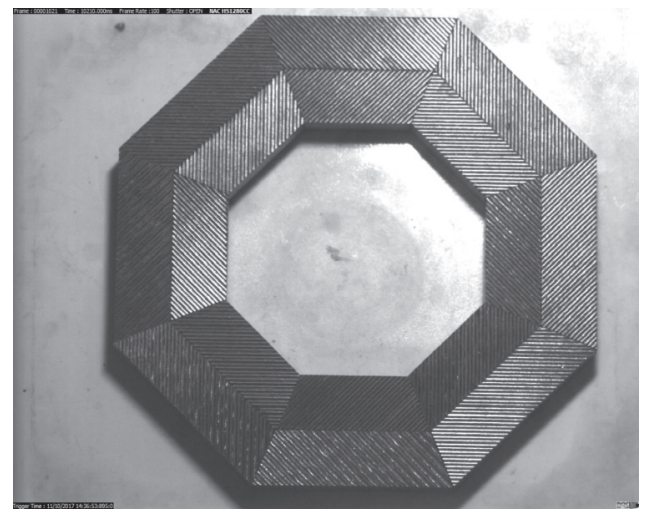

VIDEO 3. A video showing $0.47-\mathrm{mm}$-high by 1 -mm-wide sawtooth triangles (ratchet angle $\beta=25^{\circ}$ ) in a herringboneratchet structure manufactured into an octagon shape.

of the substrate as it approaches the center line. A comparative estimate of the centering distance of a droplet on different herringbone-ratchet geometries can be made from the ratio $F_{p} / v_{t}^{2}$. Although the propulsion force on a $\beta=$ $15^{\circ}$ ratchet is larger than the $\beta=42^{\circ}$ ratchet, the acceleration and terminal velocity of the droplet are also larger. Considering that the terminal velocity and propulsion force are larger on the $\beta=15^{\circ}$ compared to the $\beta=42^{\circ}$ ratchet by a factor of approximately 2 (Fig. 6 and Table I), a droplet on a $\beta=15^{\circ}$ ratchet would have a larger centering distance $d_{c}$ from Eq. (8), as can be seen in Videos 1 and 2.

The simulation of a $40 \mu 1$ droplet on these designs [using Eqs. (5) and (6)] agrees with the aforementioned travel and the centering and damped oscillation characteristics of motion on a herringbone ratchet are shown in Fig. 9. A droplet deposited off center on a $\beta=42^{\circ}$ ratchet demonstrates a better centering capability at the cost of travel speed.
To further demonstrate the ability to control the droplet direction, we create an octagon consisting of 16 angled, 0.4 -mm-high by 1 -mm-wide ratchet $\left(\beta=22^{\circ}\right)$ triangles on blocks, configured to form a herringbone-ratchet ring. Video 3 shows the octagon shape heated, with droplets dispensed on the surface. As can be seen, the droplet path can be controlled by changes in the center line of the combined herringbone-ratchet octagonal shape.

\section{B. Centered droplet movement}

The displacement profiles for droplets of different volumes released on the center line of the $\beta=42^{\circ}$ structure can be seen in Fig. 10. The droplets, once centered, travel at $200-250 \mathrm{~mm} \mathrm{~s}^{-1}$ along the center line, depending on the droplet volume. Larger droplets accelerate more quickly than smaller droplets and the maximum velocity appears to increase slightly with increasing droplet volume. The droplets are tested at both $250^{\circ} \mathrm{C}$ and $320^{\circ} \mathrm{C}$, with the droplets traveling at higher speeds for the higher temperature. The terminal velocity of a droplet on this herringbone-ratchet design is larger compared to the equivalent ratchet-only design, which provides speeds of up to $150 \mathrm{~mm} \mathrm{~s}^{-1}$. The ability to better control the position of the droplets at high velocities is a major advantage of the herringbone-ratchet design.

In the case of the $\beta=15^{\circ}$ structure, the lower ratchet height results in higher velocities (as can be seen in Fig. 11 and Video 2). Droplets travel with maximum experimental velocities of $240-330 \mathrm{~mm} \mathrm{~s}^{-1}$ depending on the droplet volume, compared to $200-250 \mathrm{~mm} \mathrm{~s}^{-1}$ for the higher ratchet angles. However, in this case the trend for the droplet volume and the terminal velocity is opposite to that observed in the $\beta=42^{\circ}$ structure, with larger-volume droplets traveling more slowly than smaller ones.

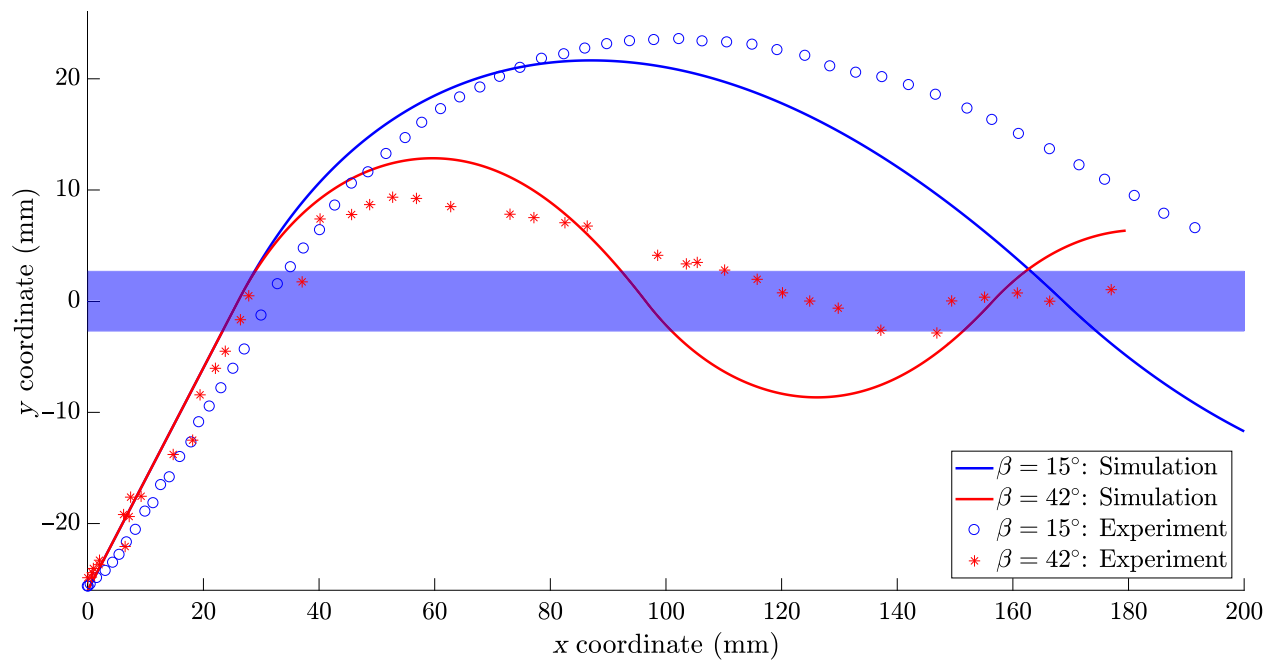

FIG. 9. A comparison of the simulated movement of $40 \mu 1$ droplets on different designs. $x$ is the travel direction and $y$ is the direction across the herringbone-ratchet structure. The droplet is released from $(x, y)=\left(\begin{array}{lll}0 & \mathrm{~mm},-25 \mathrm{~mm}\end{array}\right)$ at $t=0 \mathrm{~s}$. The shaded blue region shows the droplet diameter. 


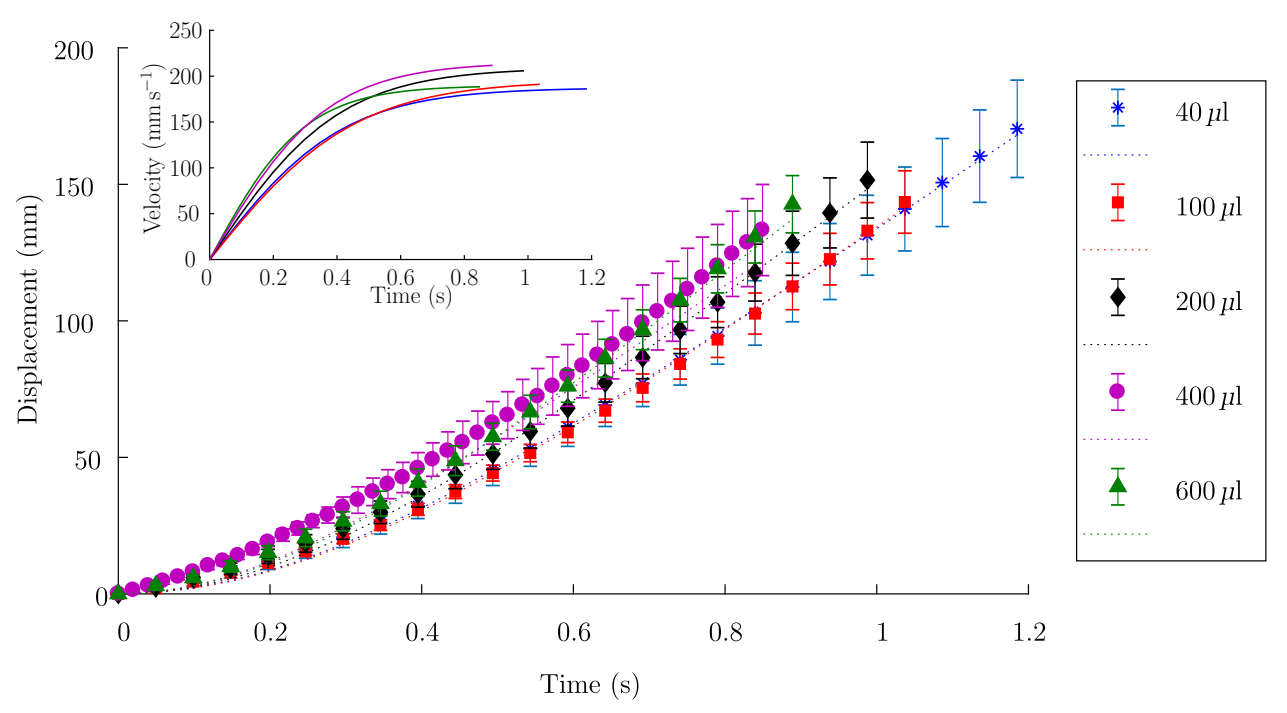

FIG. $\quad$ 10. Experimentally observed average- $x$-displacement (center-line) profiles of water droplets of different volumes released on the center line of a $\beta=42^{\circ}$ herringbone ratchet at $250^{\circ} \mathrm{C}$. The dotted lines are the fit curves of Eq. (3) on the experimental data, which are used to obtain $v_{t}$ and $\tau$ for each droplet volume. The inset shows the velocity vs time curves, represented by Eq. (2), using the values of $v_{t}$ and $\tau$. For more information, see Table I.

To investigate the trends of the terminal velocity $\left(v_{t}=\sqrt{F_{p} / c_{i}}\right.$ against the droplet volume, we observe the variation of $F_{p}$ with droplet radius $R$, considering $c_{i} \propto$ $R^{2}$ [12]. The shear force from the vapor flow is given as $F_{p} \propto R^{3} / h^{3}$ [13]. In the case of lower ratchet heights [Fig. 12(a)], the vapor-layer thickness can be assumed to be uniform across the entire liquid-vapor interface [13]. In such a case, the average vapor-layer thickness is estimated from two equations. The first is an energy-balance equation, where the heat transfer across the vapor layer is converted into the latent heat of vaporization. The second equation balances the pressure from the exiting vapor with the weight of the levitating droplet. These equations result in $h=\sqrt{b R}$, where $b=\sqrt{3 k \eta \Delta T /\left(4 L \rho_{a} \rho_{w} g l_{c}\right)}$ [13]. The above expressions give $F_{p} \propto R^{3 / 2}$, resulting in $v_{t} \propto R^{-1 / 4}$; for the $\beta=15^{\circ}$ ratchet $v_{t} \propto R^{-0.15}$ from the experiments.
In the case of larger ratchet heights, the liquid-vapor interface does not interact significantly with the ratchet surface, as shown in Fig. 12(b). In this case, the vaporlayer thickness providing the shear force is predominantly identified by the ratchet height and the deformation of the liquid-vapor interface. Both of these factors remain mostly independent of the vapor flow velocity and are identified by the surface tension and the geometry of the ratchet. Therefore, as $h$ remains independent of $R, F_{p} \propto R^{3}$, resulting in $v_{t} \propto \sqrt{R}$. The above explanation indicates a terminal velocity that increases with the droplet volume, similar to what is observed experimentally for the $\beta=15^{\circ}$ structure. However, it is important to note that the scaling observed from the experiments does not agree with the 0.5 exponent; from experiments, the exponent is approximately 0.1 . This difference in scaling might be because of interaction of the

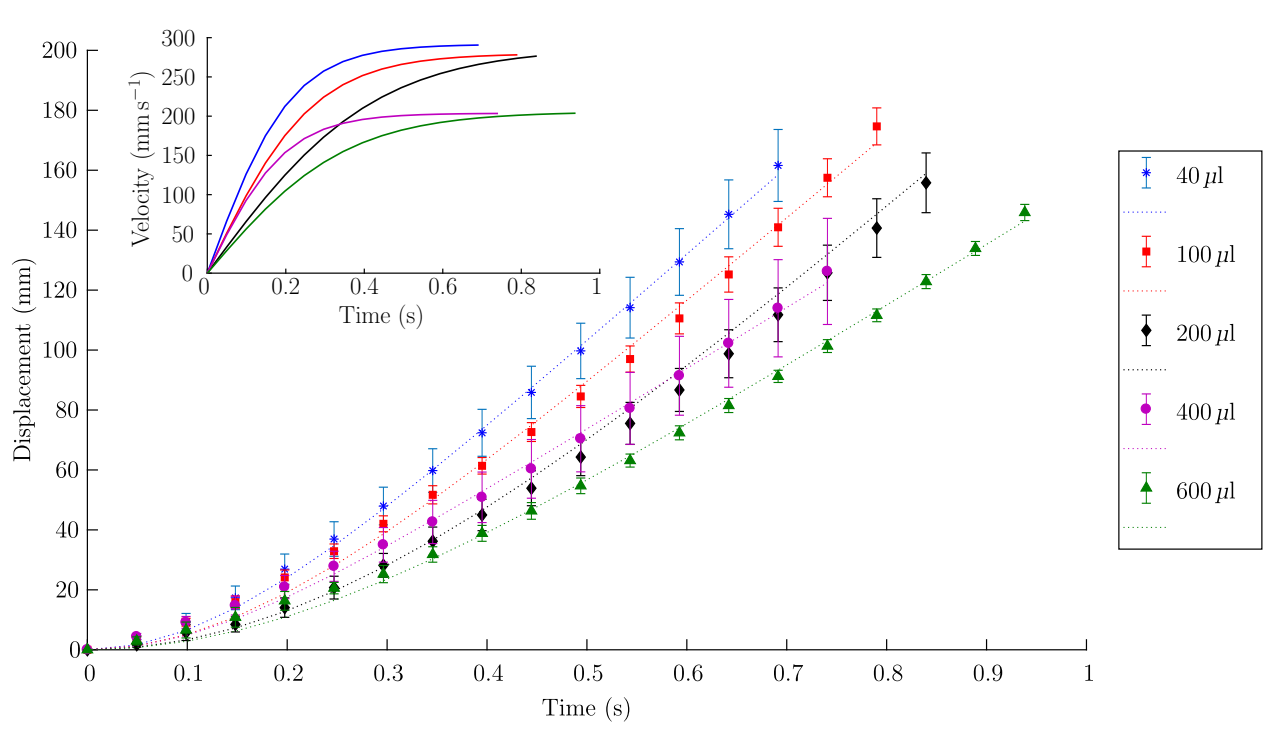

FIG. 11. Experimentally observed average- $x$-displacement (center-line) profiles of water droplets of different volumes released on the center line of a $\beta=15^{\circ}$ herringbone ratchet at $250^{\circ} \mathrm{C}$. The dotted lines are the fit curves of Eq. (3) on the experimental data, which are used to obtain $v_{t}$ and $\tau$ for each droplet volume. The inset shows the velocity vs time curves, represented by Eq. (2), using the values of $v_{t}$ and $\tau$. For more information, see Table I. 
(a)

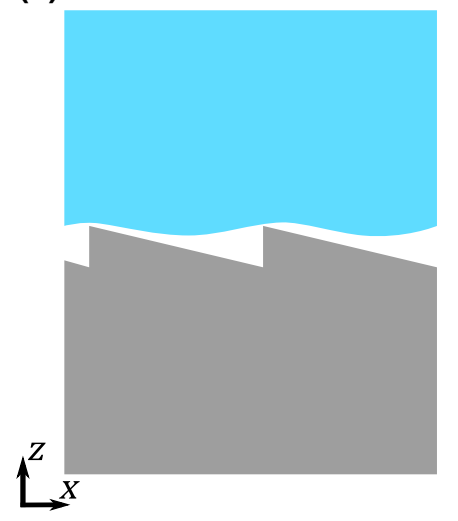

(b)

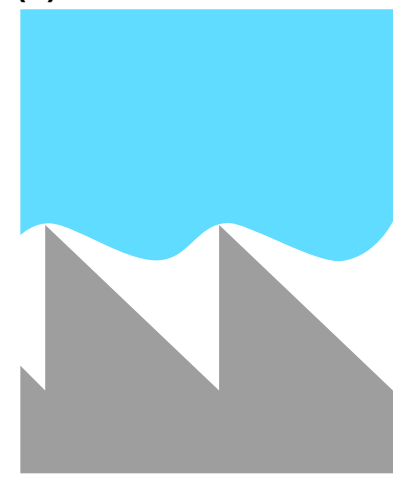

FIG. 12. A pictorial representation of the liquid-vapor interface over (a) a $0.25 \mathrm{~mm} \times 1 \mathrm{~mm}$ ratchet and (b) a $1 \mathrm{~mm} \times 1 \mathrm{~mm}$ ratchet.

vapor layer with the ratchet tip, which is not considered in the aforementioned scaling.

\section{CONCLUSION}

In this work, we introduce the concept of negative feedback to enable Leidenfrost levitation and stable lowfriction droplet transportation along user-defined paths. In our implementation, we design a structure with a smalllength-scale ratchet on a macroscale herringbone structure. The herringbone defines a center-line direction and the ratchet provides propulsion and a feedback force stabilizing motion along the center line. The herringbone-ratchet design is optimized for droplet centering or droplet speed, with speeds of up to $330 \mathrm{~mm} \mathrm{~s}^{-1}$ on a herringbone ratchet with a ratchet angle of $15^{\circ}$, compared to the fastest speed on a ratchet of $350 \mathrm{~mm} \mathrm{~s}^{-1}$ on a ratchet with an angle of $15^{\circ}$. The greater droplet control of a herringbone ratchet leads to the ability to maneuver droplets around more complex paths. This means that the Leidenfrost effect can now provide a viable droplet-control mechanism.

\section{ACKNOWLEDGMENTS}

We are grateful to EPSRC for funding under Grants No. EP/L026899/1, No. EP/L026619/1, No. EP/L026341/1, and No. EP/P005896/1.

\section{APPENDIX: SIMULATION MESH CONVERGENCE}

The optimum mesh size for the simulations (detailed in Sec. IIB) is identified by performing a mesh convergence analysis. The simulation domain shown in Fig. 5(c) is meshed with different mesh sizes and a relativeconvergence parameter is calculated as follows on the

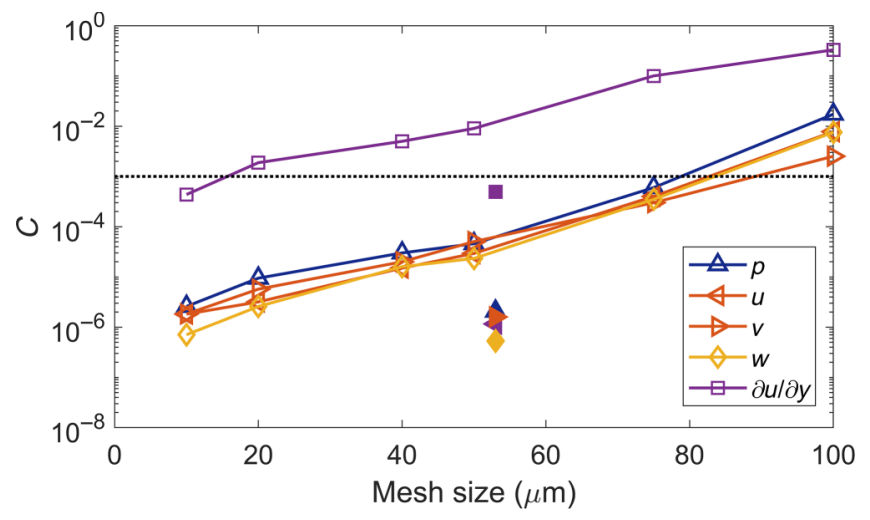

FIG. 13. The mesh convergence plot for the pressure field, the velocity field, and the $u$ velocity field gradient in the $y$ direction $(\partial u / \partial y)$. The filled markers represent the $C$ values for the chosen mesh size.

obtained velocity field:

$$
C=\sqrt{\frac{\Sigma\left(r-r_{\mathrm{ref}}\right)^{2}}{\Sigma r_{\mathrm{ref}}^{2}}} .
$$

Here, $r$ refers to the pressure and velocity field variables $(p, u, v, w$, and $\partial u / \partial y)$ and $r_{\text {ref }}$ refers to the corresponding variable for the reference mesh with maximum element size of $5 \mu \mathrm{m}$. It is important to note that this smallest mesh size is an order of magnitude smaller than the assumed vapor-layer thickness of $50 \mu \mathrm{m}$. The variation of $C$ with the different mesh sizes is shown in Fig. 13.

The simulation domain is meshed such that the corresponding value of $C$ lies below the $1 \times 10^{-3}$ threshold marked in Fig. 13. This threshold value ensures accuracy of the obtained solution while reducing the computational time of the simulation. The chosen mesh has different element sizes on different boundaries of the domain (Fig. 5): boundary 1 with $10 \mu \mathrm{m}$ and boundaries 2 and 3 with $100 \mu \mathrm{m}$ - the rest of the domain is meshed with $50-\mu \mathrm{m}$ sized tetrahedral elements. The $C$ values of the flow-field variables for this mesh size are indicated with filled markers in Fig. 13.

[1] C. Wares, On the fixation of water in diverse fire (A translation from J.G. Leidenfrost's: A tract about some qualities of common water, 1756), Int. J. Heat Mass Trans. 9, 1153 (1966).

[2] I. Vakarelski, J. Marston, D. Chan, and S. Thoroddsen, Drag Reduction by Leidenfrost Vapor Layers, Phys. Rev. Lett. 106, 214501 (2011).

[3] A. Biance, C. Clanet, and D. Quéré, Leidenfrost drops, Phys. Fluids 15, 1632 (2003). 
[4] N. R. Geraldi, G. McHale, B. B. Xu, G. G. Wells, L. E. Dodd, D. Wood, and M. I. Newton, Leidenfrost transition temperature for stainless steel meshes, Mater. Lett. 176(Supp. C), 205 (2016).

[5] K. Piroird, C. Clanet, and D. Quéré, Magnetic control of Leidenfrost drops, Phys. Rev. E 85, 056311 (2012).

[6] Y. Wang, and B. Bhushan, Liquid microdroplet sliding on hydrophobic surfaces in the presence of an electric field, Langmuir 26, 4013 (2010).

[7] B. T. Ng, Y. M. Hung, and M. K. Tan, Acousticallycontrolled Leidenfrost droplets, J. Colloid Interf. Sci. 465(Supp. C), 26 (2016).

[8] L. E. Dodd, D. Wood, N. R. Geraldi, G. G. Wells, G. McHale, B. B. Xu, S. Stuart-Cole, J. Martin, and M. I. Newton, Low friction droplet transportation on a substrate with a selective Leidenfrost effect, ACS Appl. Mater. Inter. 8, 22658 (2016).

[9] H. Linke, B. J. Alemán, L. D. Melling, M. J. Taormina, M. J. Francis, C. C. Dow-Hygelund, V. Narayanan, R. P. Taylor, and A. Stout, Self-Propelled Leidenfrost Droplets, Phys. Rev. Lett. 96, 154502 (2006).

[10] G. Dupeux, M. L. Merrer, G. Lagubeau, C. Clanet, S. Hardt, and D. Quéré, Viscous mechanism for Leidenfrost propulsion on a ratchet, Europhys. Lett. 96, 58001 (2011).

[11] T. Cousins, R. Goldstein, J. Jaworski, and A. Pesci, A ratchet trap for Leidenfrost drops, J. Fluid Mech. 696, 215 (2012).
[12] D. Soto, G. Lagubeau, C. Clanet, and D. Quéré, Surfing on a herringbone, Phys. Rev. Fluids 1, 013902 (2016).

[13] G. Lagubeau, M. L. Merrer, C. Clanet, and D. Quéré, Leidenfrost on a ratchet, Nat. Phys. 7, 395 (2011).

[14] Z. Jia, M. Chen, and H. Zhu, Reversible self-propelled Leidenfrost droplets on ratchet surfaces, Appl. Phys. Lett. 110, 091603 (2017).

[15] A. Würger, Leidenfrost Gas Ratchets Driven by Thermal Creep, Phys. Rev. Lett. 107, 164502 (2011).

[16] M. Mrinal, X. Wang, and C. Luo, Self-rotation-induced propulsion of a Leidenfrost drop on a ratchet, Langmuir 33, 6307 (2017).

[17] T. Baier, G. Dupeux, S. Herbert, S. Hardt, and D. Quéré, Propulsion mechanisms for Leidenfrost solids on ratchets, Phys. Rev. E 87, 021001 (2013).

[18] G. G. Wells, R. Ledesma-Aguilar, G. McHale, and K. Sefiance, A sublimation heat engine, Nat. Commun. 6, 6390 (2015).

[19] D. Quéré, Leidenfrost dynamics, Annu Rev. Fluid Mech. 45, 197 (2013).

[20] A. Bouillant, T. Mouterde, P. Bourrianne, A. Lagarde, C. Clanet, and D. Quéré, Leidenfrost wheels, Nat. Phys. 14, 1188 (2018).

[21] P.-G. de Gennes, F. Brochard-Wyart, and D. Quéré, Capillarity and Wetting Phenomena (Springer-Verlag, New York, 2004). 